\title{
Meningoencefalitis chagásica en un paciente con infección por VIH/SIDA con sobrevida a tres años: Caso clínico
}

\author{
Olga López M.
}

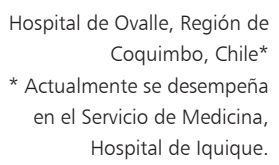

Recibido: 3 de diciembre de 2008 Aceptado: 1 de diciembre de 2009

Correspondencia a: Olga López M lopezolga@gmail.com

\author{
Three-year survival of a patient with HIV and chagasic meningoencephalitis: \\ Case report
}

Immunocompromised patients as those with renal transplant, hematological neoplasia or cancer and HIV/AIDS infection can suffer acute reactivation of Chagas disease. Central nervous system (CNS) evolvement (cerebral tumor or chagoma and diffuse meningoencephalitis) is similar to other opportunistic infections that present with cerebral expansive processes like toxoplasmosis or CNS primary lymphoma. Survival is infrequent, depending on antiparasitic therapy and early starting antiretroviral therapy. The case of an HIV/AIDS positive patient that evolved with a chagasic meningoencephalitis and improved after beginning early antiparasitic therapy and antiretroviral therapy antirretroviral is described.

Key words: Chagas disease, Trypanosoma cruzi, acquired immunodeficiency syndrome, human immunodeficiency virus, meningoencephalitis.

Palabras clave: Enfermedad de Chagas, Trypanosoma cruzi, Síndrome de inmunodeficiencia adquirida, virus de inmunodeficiencia humana, meningoencefalitis.

\section{Introducción}

La enfermedad de Chagas es una zoonosis parasitaria hemática y tisular, endémica de Latinoamérica y causada por el protozoo Trypanosoma cruzi ${ }^{1}$. Su ciclo vital presenta diferentes estadios: amastigote (intracelular), epimastigote (intestino medio del vector y en cultivo), tripomastigote (sanguíneo), y tripomastigote metacíclico (intestino posterior del vector) ${ }^{1-4}$. El amastigote se puede encontrar en tejidos comprometidos en la fase crónica visceral, en tanto que el tripomastigote se presenta en sangre y en líquido cefalorraquídeo.

En Chile el vector natural es Triatoma infestans ${ }^{1,2}$ (conocido en Chile, Argentina y Uruguay como vinchuca). La transmisión no vectorial está constituida por la vía vertical, transfusión sanguínea, trasplante de órganos sólidos, accidentes de laboratorio, transmisión alimentaria (ingesta de alimentos contaminados con el parásito) y drogadicción endovenosa ${ }^{1,2,5}$.

En nuestro país predominó históricamente la transmisión vectorial; sin embargo, las campañas de erradicación del vector del ambiente domiciliario, junto con los terremotos que destruyeron muchos de los ranchos chagásicos (casas de adobe infestadas por vinchucas), permitieron que la Organización Panamericana de la Salud (OPS) declarara interrumpida la transmisión vectorial en nuestro país ${ }^{1,3,4}$.
La enfermedad de Chagas tiene curso crónico en inmunocompetentes y oportunista en inmunodeprimidos ${ }^{1,26,7}$. Cursa en etapas aguda, crónica latente y crónica visceral. Durante la fase aguda, si la transmisión es mediada por vinchucas, puede aparecer un chagoma de inoculación. En esta etapa se observa menos frecuente miocarditis, neumonitis y meningoencefalitis. Posteriormente sigue una fase crónica latente que dura años o décadas. Finalmente, en algunos pacientes, $T$. cruzi anida en tejidos, con mayor tropismo por el tubo digestivo y corazón, desarrollando mega vísceras. La muerte se presenta sólo en las fases aguda y crónica visceral ${ }^{1,2}$.

En estados de inmunodepresión como leucemia ${ }^{8}$, trasplante renal o cardíaco ${ }^{9-11}$ e infección por $\mathrm{VIH} / \mathrm{SID}^{5,6,12,13}$, la enfermedad de Chagas se manifiesta en forma atípica, tanto en la fase aguda como en la reactivación de la fase crónica $^{1,3,14,15}$, siendo eventualmente fatales sin tratamiento oportuno $^{2,11,15,16}$. La reactivación tiene manifestaciones especiales, con mayor frecuencia el compromiso del sistema nervioso central (SNC) ${ }^{3,4,7-22}$ y la miocarditis aguda ${ }^{1,8,12,17,18}$. En el SNC adopta dos formas: meningoencefalitis con tripomastigotes en líquido cefalorraquídeo (LCR) ${ }^{11,14}$ o tumor conocido como chagoma ${ }^{8,15,17-20}$ que consiste en nidos de amastigotes asociados a encefalitis necrotizante multifocal con abscesos ${ }^{1,2,8,16,21}$. En las imágenes cerebrales, el chagoma es indistinguible de otras infecciones oportunistas como la toxoplasmosis y del linfoma pri- 
mario de $\mathrm{SNC}^{1,2,13,19}$, y cursa con alta mortalidad ${ }^{8,14-19}$. En 1990, Del Castillo comunicó la primera asociación de infección por VIH y enfermedad de Chagas ${ }^{15}$. En Chile, Oddó y cols. y Labarca y cols. describieron en 1992 dos casos de enfermedad de Chagas con compromiso del SNC en pacientes en etapa SIDA, con evolución fatal ${ }^{17,19}$. Córdova y cols, comunicaron una mortalidad de $79 \%$ con una mediana de sobrevida de 21 días $^{10}$. En la Tabla 1 se muestra un resumen de la casuística publicada.

A continuación se reporta un caso de meningoencefalitis chagásica en un paciente con infección por VIH/SIDA que inició tratamiento precoz exitoso y con sobrevida a tres años.

\section{Caso clínico}

Varón de 36 años oriundo de Andacollo que vivió siempre en la Región de Coquimbo, creció en casa de adobe y trabajaba como folclorista. Algunos de sus familiares tenían antecedentes de enfermedad de Chagas con megavísceras. Recordaba que en su infancia había jugado con vinchucas. Negaba haber tenido un chagoma de inoculación, usado drogas endovenosas o haber recibido transfusiones sanguíneas.

Tres meses antes de su hospitalización, presentó un cuadro de baja de peso progresiva, diarrea crónica, disfagia y postración, motivo por el cual se le realizó serología para VIH, que resultó positiva y fue confirmada por el ISP. Una semana antes de su hospitalización se agregó fiebre, cefalea intensa, convulsiones, alteración de memoria y pérdida de control de esfínteres. Al examen físico destacó emaciación, fiebre, alteración de la memoria, reflejos osteotendineos conservados y dudosa rigidez de nuca. No había déficit focal neurológico. En el estudio del laboratorio general sólo destacó anemia leve. En el fondo de ojos se pesquisó alteraciones retinianas compatibles con retinopatía por VIH. La endoscopia digestiva alta demostró lesiones sugerentes de candidiasis esofágica. La serología IgM e IgG para Toxoplasma gondii resultaron negativas. El estudio de LCR (Tabla 2) evidenció la presencia de tripomastigotes (Figura 1), las determinaciones de tinta china, adenosin deaminasa (ADA), VDRL, cultivo corriente y células neoplásicas en LCR resultaron negativas. No se disponía de panel de estudio para otras etiologías de encefalitis. No se realizó electroencefalograma. La serología de Chagas por técnica ELISA e IFI fueron positivas, esta última en títulos de $\operatorname{IgG} 1 / 160$. Se descartó compromiso de órganos blancos habituales para megavísceras. La tomografía axial computada (TAC) de cerebro mostró lesiones hipodensas en la sustancia blanca en ambos hemisferios cerebrales (Figura 2-A). En base a todos estos hallazgos se diagnosticó meningoencefalitis por reactivación de enfermedad de Chagas.
Se inició nifurtimox $200 \mathrm{mg}$. cada ocho horas junto con fenitoína y fue evaluado quincenalmente con hemograma, creatininemia y pruebas de función hepática. Se difirió en dos semanas el comienzo de la terapia antiretroviral

Tabla 1. Casos publicados en la literatura médica de meningoencefalitis chagásica en pacientes con infección por VIH/SIDA

\begin{tabular}{|c|c|c|c|c|}
\hline Autores & Año & Casos & Tratamiento & Sobrevida \\
\hline Del Castillo et al. & 1990 & 1 & Nifurtimox, cirugía & Más de 3 meses \\
\hline Labarca et al. & 1992 & 2 & No & No \\
\hline Gluckstein et al. & 1992 & 1 & No & No \\
\hline Ferreira et al. & 1992 & 1 & No & No \\
\hline Gallo et al. & 1992 & 1 & Beznidazol & 2 meses \\
\hline Oddó et al. & 1992 & 1 & Beznidazol & Más de 6 meses \\
\hline Rosemberg et al. & 1992 & 1 & No & No \\
\hline Rocha et al. & 1993 & 1 & No & No \\
\hline Nishioka et al. & 1993 & 1 & Beznidazol & Desconocida \\
\hline Metze & 1993 & 1 & Beznidazol & Desconocida \\
\hline Pimentel & 1996 & 1 & Beznidazol & 1 mes \\
\hline Montero et al. & 1998 & 1 & Nifurtimox & No \\
\hline Gutierrez et al. & 1999 & 1 & Beznidazol & No \\
\hline Martins et al. & 2002 & 1 & Beznidazol & No \\
\hline Lages-Silva & 2002 & 1 & Beznidazol & Sí, con secuelas \\
\hline De Oliveira et al. & 2002 & 1 & No & No \\
\hline Yoo et al. & 2004 & 1 & Nifurtimox & No \\
\hline Madalosso et al. & 2004 & 1 & Beznidazol & No \\
\hline Ferreira et al. & 2004 & 1 & No & No \\
\hline Valerga et al. & 2006 & 1 & Beznidazol & No \\
\hline Frías et al. & 2006 & 1 & No & No \\
\hline Corti et al. & 2006 & 1 & Beznidazol & Sí, prolongada \\
\hline Córdova et al. & 2008 & 15 & Beznidazol & $3 / 15$ \\
\hline
\end{tabular}

Tabla 2. Meningoencefalitis chagásica. Características del LCR al momento del diagnóstico y a las dos semanas de tratamiento antiparasitario

\begin{tabular}{|lll|}
\hline Parámetros de laboratorio & Día $\mathbf{0}$ & Día $\mathbf{1 4}$ \\
\hline Goteo & Rápido & Lento \\
\hline Turbidez & + & - \\
\hline Glucosa $(\mathrm{mg} / \mathrm{dl})$ & 0,48 & 0,7 \\
\hline Proteínas $(\mathrm{ml} / \mathrm{dl})$ & 0,84 & No se realizó \\
\hline Células $/ \mathrm{mm}^{3}$ & 50 & 1 \\
\hline Polimorfonucleares & $90 \%$ & - \\
\hline Mononucleares & $10 \%$ & - \\
\hline
\end{tabular}


Figura 1. Tripomastigote en líquido cefalorraquídeo con tinción de Giemsa 40X.
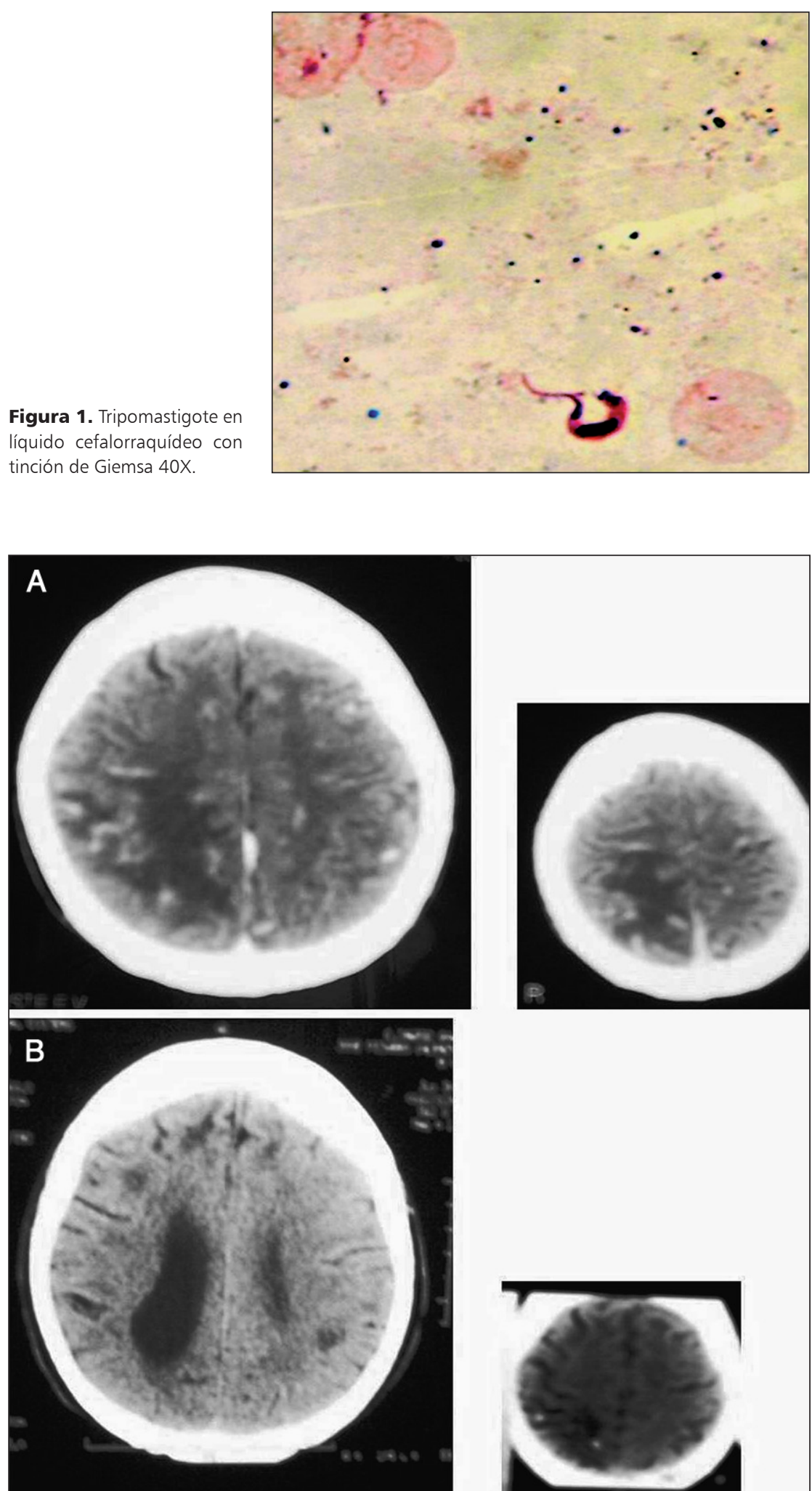

Figura 2. Tomografía axial computada de cerebro sin contraste al diagnóstico y al año de evolución. (A) Se observan lesiones hipodensas difusas en la sustancia blanca de ambos hemisferios cerebrales. (B) Persisten escasas lesiones hipodensas en la sustancia blanca de la región occipital con algunas calcificaciones.
(TARV) para evitar un síndrome de reconstitución inmune. Al tercer día de iniciada la TARV con zidovudina/ lamivudina en coformulado y efavirenz, presentó un episodio sicótico agudo con alucinaciones visuales y auditivas, que revirtieron al reemplazar el efavirenz por nevirapina.

A las dos semanas de iniciada la TARV se pudo realizar las determinaciones basales de carga viral de 44.000 copias de ARN viral/ml (Nuclisens HIV-1 QTTM, Biomérieux) y recuento de LTCD4 de 135 céls $/ \mathrm{mm}^{3}$. Al mes de tratamiento recuperó la memoria de corto y de mediano plazo, se constató leve dismetría y paresia de la extremidad inferior derecha. Al quinto mes presentó hepatomegalia leve con discreta elevación de las transaminasas, decidiéndose mantener el tratamiento con monitorización estricta. A los 7 meses de terapia para ambas condiciones, tenía recuento de LTCD4 de 256 céls $/ \mathrm{mm}^{3} \mathrm{y}$ una carga viral indetectable. Durante el tratamiento con nifurtimox no se dispuso de técnicas de biología molecular para la detección de $T$. cruzi. Una vez superada la paresia y la dismetría, y con LTCD4 mayor a 200 céls $/ \mathrm{mm}^{3}$ se decidió suspender la terapia antiparasitaria.

Al año de evolución, se efectuó la reacción de polimerasa en cadena (RPC) de tipo cualitativa en sangre para T. cruzi, la cual fue negativa, por lo que no se dio nuevo tratamiento con nifurtimox. La TAC de cerebro demostró remisión de las lesiones de sustancia blanca, aparición de calcificaciones múltiples en la región occipital derecha (Figura 2-B), manteniéndose con fenitoína. La evaluación por neurología, descartó otras causas de calcificaciones del SNC.

Actualmente, se encuentra en TARV exitosa y no ha vuelto a presentar síntomas neurológicos. A tres años del compromiso meníngeo parasitario, el paciente desarrolla una vida normal similar a la previa a su cuadro.

\section{Discusión}

Las formas más graves de presentación de la enfermedad de Chagas con compromiso del SNC se dan en pacientes que adquirieron la enfermedad parasitaria posterior al contagio con $\mathrm{VIH}^{2}$. En el $85 \%$ de las de meningitis chagásicas agudas se puede encontrar T. cruzi en el $\mathrm{LCR}^{1,5,8-11,21}$. Las técnicas diagnósticas directas no requieren confirmación ya que brindan un diagnóstico de certeza $^{1,2}$. Los métodos indirectos junto con alteraciones de SNC pueden ayudar al diagnóstico ${ }^{7}$. Las lesiones infratentoriales asociadas a compromiso supratentorial en la TAC de encéfalo son sugerentes, aunque las imágenes normales no descartan esta complicación ${ }^{2,5,7,8,11,13,17,20}$. Si persisten dudas puede plantearse una biopsia estereotáxica en la que se observará una encefalitis extensa multifocal, necrohemorrágica, con angeítis obliterante y amastigotes 
en células gliales, macrófagos y células endoteliales ${ }^{1,7,22}$. Un enfoque terapéutico útil es el propuesto por Auger y cols (Figura 3) ${ }^{7}$.

Es anecdótica la sobrevida y se da en aquellos casos donde existe la sospecha clínica e inicio precoz del tratamiento antiparasitario, asociado a TARV ${ }^{1,5,10}$.

Los fármacos tripanomicidas clásicos utilizados en el tratamiento son nifurtimox y benznidazol. Nifurtimox es un análogo de nitrofuranos, con efecto tripanomicida y actúa contra las formas de amastigote y tripamastigote de T. cruzi. La dosis terapéutica recomendada de nifurtimox es 8 a $10 \mathrm{mg} / \mathrm{kg} /$ día, fraccionado cada 8 horas. Los efectos secundarios se presentan en $30 \%$ de los casos, especialmente en adultos. Puede producir anorexia, pérdida de peso, manifestaciones gastrointestinales como náuseas, vómitos, dolor abdominal, diarrea, dermatitis y compromiso del SNC con insomnio, alucinaciones, parestesias y psicosis ${ }^{1,2,11,23}$. La anorexia puede ser marcada y se debe ajustar la dosis si el paciente empieza a bajar mucho de peso.

Benznidazol también es un antiparasitario tripanomicida, que actúa uniéndose en forma covalente a los intermediarios de la nitrorreducción con los componentes del parásito, ADN, lípidos y proteínas. La dosis terapéutica recomendada es: $5 \mathrm{mg} / \mathrm{kg} / \mathrm{día}$, fraccionado cada 12 horas. Los efectos adversos se dividen en tres tipos: dermatológicos con erupción cutánea que aparece entre los 7-10 días de tratamiento, edema generalizado, fiebre, adenopatías, mialgias y artralgias; depresión de la médula ósea y compromiso neurológico con polineuropatía, parestesia y polineuritis periférica ${ }^{1,2,11,23}$. De estos medicamentos, sólo nifurtimox está disponible en Chile. Debiese administrarse antiparasitarios a dosis plena por noventa días o más ${ }^{20}$, seguido de una terapia supresiva o de mantención, hasta que el paciente mejore su recuento de LTCD4 con la terapia antirretroviral. Una alternativa sería prescribir nifurtimox en forma trisemanal ${ }^{1}$.

Córdova y cols, han reportado parasitemia, poco frecuente a largo plazo $^{10}$. Los pocos reportes de este cuadro en Chile se deberían al desconocimiento de su existencia $^{12,18,19}$. No está claro el significado de la parasitemia persistente y su implicancia en el pronóstico en la progresión de la enfermedad de Chagas en estos casos 5.

En pacientes infectados por VIH/SIDA, con meningoencefalitis, con o sin lesiones focales, se debe considerar en el diagnóstico diferencial la reactivación aguda de la enfermedad de Chagas, sobre todo en países de alta endemia $^{7,10,12,18,19}$. No existe consenso respecto a la duración de la terapia o acerca de esquemas de profilaxis secundaria ${ }^{20}$.

En resumen, la publicación de ese caso tiene como finalidad incorporar en los médicos clínicos la sospecha de reactivación de la enfermedad de Chagas, en el diagnóstico diferencial de las meningoencefalitis de líquido claro en pacientes con infección por VIH/SIDA. La disponibilidad

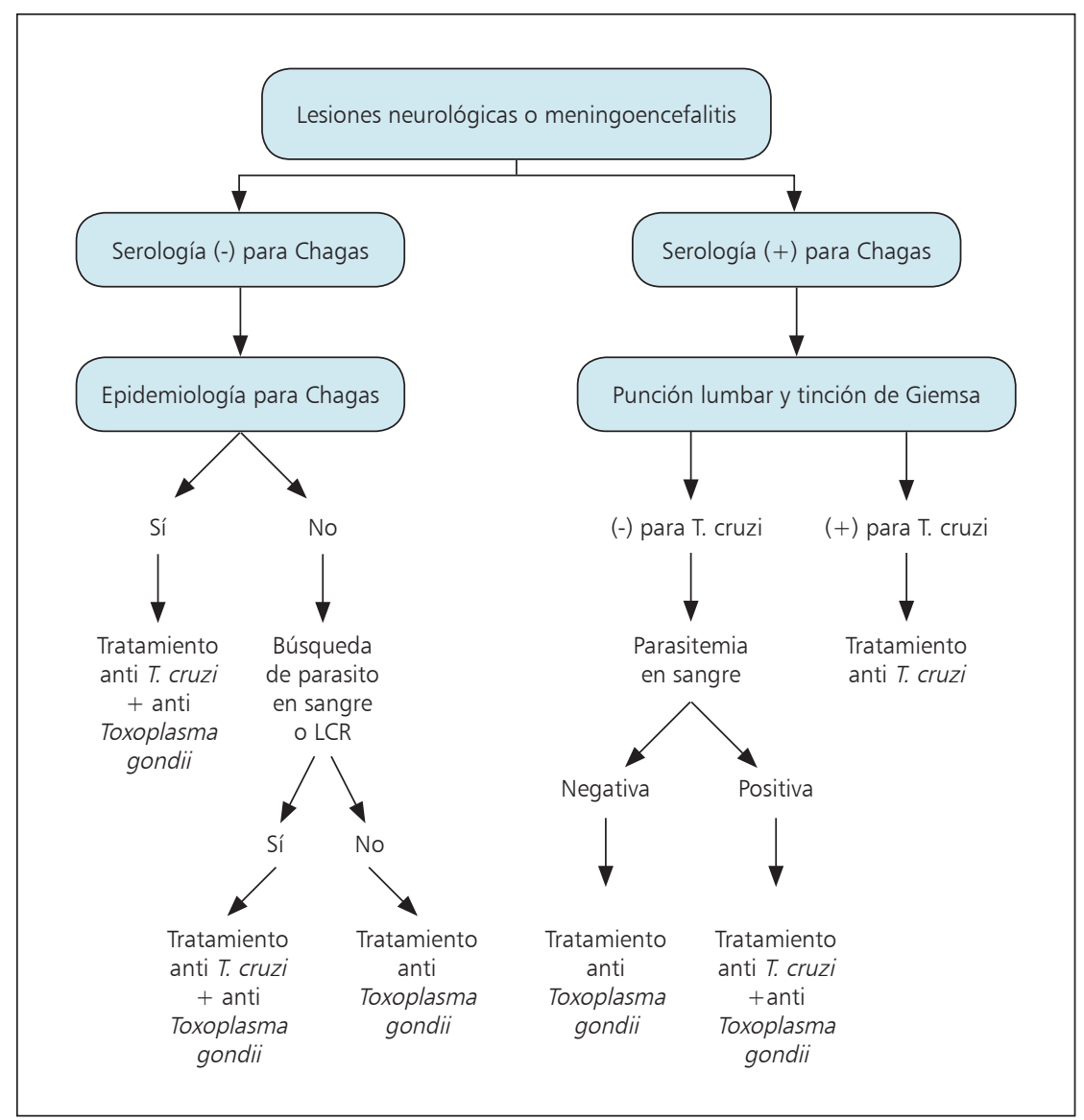

Figura 3. Algoritmo de diagnóstico y tratamiento de las lesiones neurológicas o meningoencefalitis en el SIDA en regiones endémicas de la enfermedad de Chagas propuesto por Auger y cols (ref 24).

de nifurtimox brinda una oportunidad inédita de marcar un quiebre en la evolución natural de las reactivaciones agudas en pacientes inmunocomprometidos. El paciente actualmente sigue viviendo en Andacollo en una casa de construcción sólida.

La certificación serológica de la interrupción de la transmisión vectorial de la enfermedad de Chagas en nuestro país otorgada por la OPS, implica que no se han encontrado vinchucas infestadas desde el año 2001, lo cual está respaldado por una vigilancia permanente que busca la reaparición del vector. Todo lo anterior hace poco probable que el paciente se pueda reinfectar.

En el mundo globalizado actual, es importante mantener altos niveles de sospecha y vigilancia de este cuadro clínico. Cabe destacar que Perú y Bolivia mantienen la transmisión vectorial de la enfermedad de Chagas, esto es importante de considerar dado los constantes movimientos migratorios hacia y desde la zona del Norte Grande de Chile. Por otra parte, este sector cuenta con las tasas más altas de infección por VIH/SIDA del país, condicionando 
un mayor riesgo de aparición de nuevos casos de esta patología.

Agradecimientos: A Irene Levy por la foto de tripomastigote en líquido cefalorraquídeo. A Susana Verdugo, Proyecto Fondecyt 2005 "Prevención de transmisión vertical de la Enfermedad de Chagas en el Hospital de Ovalle" por hacer posible el tratamiento con nifurtimox.

\section{Resumen}

Los pacientes inmunocomprometidos como los sometidos a trasplantes renales, con neoplasias hemato- oncológicas e infección por VIH/SIDA, pueden desarrollar reactivación aguda de la enfermedad de Chagas. El compromiso del sistema nervioso central-SNC (tumor cerebral o chagoma y meningoencefalitis difusa) es similar a otras infecciones oportunistas que cursan con procesos expansivos cerebrales como toxoplasmosis o linfoma primario del SNC. La sobrevida es poco frecuente, siendo prioritario para la buena evolución, la terapia antiparasitaria y el inicio de terapia antiretroviral. Se describe caso clínico de un paciente con infección por VIH/SIDA que cursó con meningoencefalitis chagásica con evolución satisfactoria tras el inico precoz de terapia antiparasitaria y terapia antiretroviral.

\section{Referencias}

1.- Guías Clínicas de la prevención y control de la Enfermedad de Chagas 2006. Ministerio de Salud, Chile.

2.- Consenso argentino de enfermedad de Chagas Rev Argent Cardiol 2002; 70 S: 1-87.

3.- Lorca M. Certificación serológica de la interrupción de la transmisión vectorial de la enfermedad de Chagas en Chile. Rev Méd Chile 2001; 129: 266-71.

4.- García A, Bahamonde M, Verdugo S, Correa J, Pastene C, Solari A. et al. Infección transplacentaria por Trypanosoma cruzi. Rev Méd Chile 2001; 129: 330-2.

5.- Corti M, Yampolsky C. Prolonged survival and immune reconstitution after chagasic meningoencephalitis in a patient with acquired immunodeficiency syndrome. Rev Soc Bras Med Trop 2006; 39: 85-8.

6.- Bentwich Z, Maartens G, Torten D, Altaf A. Concurrent infections and HIV pathogenesis. AIDS 2000; 14: 2071-81.

7.- Auger S, Storino R, De Rosa M, Caravello O, González M, Botaro E. et al. Chagas y SIDA, la importancia del diagnóstico precoz. Rev Argent Cardiol 2005; 73: 439-445.

8.- Corti M. AIDS and Chagas disease. AIDS Patient Care AND STDS 2000; 14: 581-7.

9.- Sartori, Ibrahim K, Nunes Westphalen E, Braz L, Oliveira O, Gakiya E, et al. Manifestations of Chagas disease
(American trypanosomiasis) in patients with HIV/ AIDS. Ann Trop Med Parasitol 2007; 101: 31-50.

10.- Córdova E, Boschi A, Ambrosioni J, Cudos C, Corti M. Reactivation of Chagas disease with central nervous system involvement in HIV-infected patients in Argentina, 1992-2007. Int J Infect Dis 2008: 12: 587-92.

11.- Yoo T, Mlikotic A, Cornford M, Beck C. Concurrent cerebral American Trypanosomiasis and toxoplasmosis in a patient with AIDS. Clin Infect Dis 2004; 39 (4): e30-4.

12.- Solari A, Saavedra H, Sepúlveda C, Oddó D, Acuña G, Labarca J, et al. Successful treatment of Trypanosoma cruzi encephalitis in a patient with hemophilia and AIDS. Clin Infect Dis 1993; 16: 255-9.

13.- Lages-Silva E, Ramirez LE, Silva-Vergara ML, Chiari E. Chagasic meningoencephalitis in a patient with acquired immunodeficiency syndrome: diagnosis, follow-up, and genetic characterization of Trypanosoma cruzi. Clin Infect Dis 2002; 34: 118-23.

14.- Corona S, Amanales C, Avaria M, Colin E, Donoso S, Advis P et al. Granuloma chagásico del cerebro en un paciente con leucemia linfoblástica. Rev Méd Chile 1988; 116: 676-80.

15.- Del Castillo M, Mendoza G, Oviedo J, Pérez Blanco RP, Anselmo AE, Silva A M. AIDS and Chagas disease with central nervous system tumor-like lesion. Amer J Med 1990; 88: 693-4.

16.- Valerga M, Bases O, Martin M, Papucci T. Encefalitis multifocal en un paciente con SIDA. Enferm Infecc Microbiol Clin 2005; 23: 569-70.

17.- Rocha M, Ferreira S, Nishioka M, Silva M, Burgarelli M, Silva A. et al. Trypanosoma cruzi meningoencephalitis and miocarditis in patient with acquired immunodeficiency syndrome. Rev
Inst Med Trop S Paulo 1993; 35: 205-8.

18.- Oddó D, Casanova M, Acuña G, Ballesteros J, Morales B. Acute Chagas disease (Trypanosomiasis Americana) in acquired immunodeficiency syndrome: report two cases. Human Pathology 1992; 23: 41-4.

19.- Labarca J, Acuña G, Leiva J, Cambon G, Oddó B, Sepúlveda C, et al. Enfermedad de Chagas en el Síndrome de Inmunodeficiencia Adquirida. Casos clínicos. Rév Méd chile 1992; 120: 174-9.

20.- Guidelines for Prevention and Treatment of Opportunistic Infections in HIV-Infected Adults and Adolescents. MMWR 2009; 58( RR-4): 103-5.

21.- Madalosso G, Guedes A, Vasconcelos M, Freitas Ribeiro A, Weissmann L, Silva G, Oliveira F. et al. Chagasic meningoencephalitis: case report of a recently included AIDS-defining illness in brazil. Rev Inst Med Trop S Paulo. 2004; 46: 199-202

22.- Torrealba G, Acuña G, Tagle P, Tapia J, Huete I. Valor de la biopsia cerebral en pacientes con SIDA y lesiones expansivas cerebrales. Rev Méd Chile 1990; 118: 1367-71.

23.- Maya J, Rodríguez A, Pino A, Pabón A, Ferreira J, Pavani M, et al. Effects of buthionine sulfoximine nifurtimox and benznidazole upon trypanothione and metallothionein proteins in Trypanosoma cruzi. Biol Res 2004; 37 : 61-9. 